\title{
近 10 年英语教育硕士学位论文选题热点可视化分析
}

\section{Visual Analysis of Hot Topics in English Education Master's Thesis in Recent 10 Years}

\author{
徐庆利 \\ Qingli Xu \\ 曲阜师范大学外国语学院 中国·山东曲阜 273165 \\ Qufu Normal University College of Foreign Languages, Qufu, Shandong, 273165, China
}

\begin{abstract}
摘 要: 本研究通过知识图谱绘制软件 $\mathrm{COOC}$, 从词频、共现和聚类 3 个方面对近 10 年英语教育硕士学位论文选题热点 进行了可视化分析。研究发现，该阶段英语教育硕士选题符合国家对教育硕士专业培养的要求，主要表现为聚焦基础阶段 英语教学, 侧重开展解决教学实际问题的应用型研究, 能用相关学科理论和科学的实证研究方法来探究问题解决路径。但 同时选题也存在缺乏创新性、前沿性，对小学英语教学关注度不够等问题。
\end{abstract}

\begin{abstract}
In this study, $\mathrm{COOC}$, a knowledge mapping software, was used to visually analyze the hot topics of English master's thesis topics in recent 10 years from three aspects: word frequency, co-occurrence and clustering. It is found that the topic selection of master of English education in this stage conforms to the national requirements for the cultivation of master of Education majors, which is mainly manifested as focusing on English teaching in the basic stage, focusing on applied research to solve practical problems in teaching, and using relevant discipline theories and scientific empirical research methods to explore solutions to problems. But at the same time, there are also some problems, such as lack of innovation, frontier, and insufficient attention to primary school English teaching.
\end{abstract}

关键词：英语教育硕士；学位论文选题；热点；可视化分析

Keywords : english education master; the choice of dissertation topic; hot spot; visual analysis

基金项目: 山东省 2017 年研究生导师指导能力提升项目 “以 “应用研究能力培养”为导向的《外语教学科研方法与论文写作》 课程建设研究” (SDYY17137)

DOI : $10.36012 /$ sde.v2i11.2313

\section{1 引言}

近 10 年来, 为顺应国家和社会对高层次、专家型中小 学英语教师的需求, 英语教育硕士招生规模不断扩大, 英语 教育硕士培养质量问题也日益引起关注。目前, 研究者主要 从培养模式、专业发展能力以及学位论文 3 个方面对中国英 语教育硕士培养问题进行探讨。学位论文是教育硕士培养的 重要组成部分和总结性成果, 深入分析学位论文质量对进一 步改进和完善教育硕士培养模式具有重要意义 ${ }^{[1,2]}$ 。但目前 该领域基于数据的量化研究还较少, 而已有的量化研究由于 没有使用先进的数据分析软件, 对数据的挖掘深度不够。因
此, 论文将在前人研究的基础上, 从学位论文选题视角进一 步聚焦英语教育硕士学位论文质量问题，即采用科学计量学 研究方法, 借助新兴的知识图谱绘制软件 COOC, 对近 10 年 英语教育硕士学位论文选题热点进行深度分析, 以期为该阶 段英语教育硕士培养方向是否符合国家对专业硕士培养的 要求、紧跟国家教育改革步伐提供可靠的量化依据。

\section{2 研究设计}

\section{1 研究方法和工具}

选题是学位论文写作的第一步, 也是极其重要且具有 战略性意义的一步, 体现着论文的研究方向、研究目的以及

【作者简介】徐庆利 (1976 ), 女, 山东滕州人, 副教授, 主要从事第二语言习得、外语教学理论与实践研究。 
作者的知识视野和创新意识, 是评价论文写作质量以及研究 生培养质量的首要依据。因此, 本文聚焦于 2009-2019年， 采用科学计量学研究方法, 应用数理统计和先进的知识图谱 绘制软件 $\mathrm{COOC}$, 对该时间段英语教育硕士学位论文选题进 行基于高频词的统计和可视化分析，以探测近 10 年英语教 育硕士学位论文选题热点，揭示该阶段英语教育硕士培养取 得的成就和存在的问题。

\section{2 数据采集}

本文数据来源为中国知网硕士学位论文全文数据库, 学位年度选择为 2009-2019 年。由于题名是反映选题热点 的重要线索, 因此研究者以题名为“英语”且题名为“教 学”或含“学习”为入口检索文献, 然后对检索到的文献的 专业方向进一步核实, 剔除教育管理等非英语教育硕士以及 语言学等学术型硕士的学位论文, 最终得到符合要求的文献 12204 篇，即 12169 条题名，同时为捕捉到更有意义的关键 词，在进行数据分析时，笔者剔除了题名中含有的“研究”“探 究”“探索”等意义比较空泛的词汇。

\section{3 结果与讨论}

\section{1 基于词频的论文选题热点分析结果及讨论}

研究热点可以认为是在某个领域中学者共同关注的一 个或者多个话题, 从字面上理解, 其具有很强的时间特征 ${ }^{[3]}$ 。 对一段时间内文献题目中的高频词进行分析, 可以探测该时 间段内某学科领域研究者共同关注的热点。

图 1 为 $\mathrm{COOC}$ 软件在对词频统计分析基础上绘制出来 的近 10 年英语教育硕士学位论文选题热点高频关键词可视 化词云图。

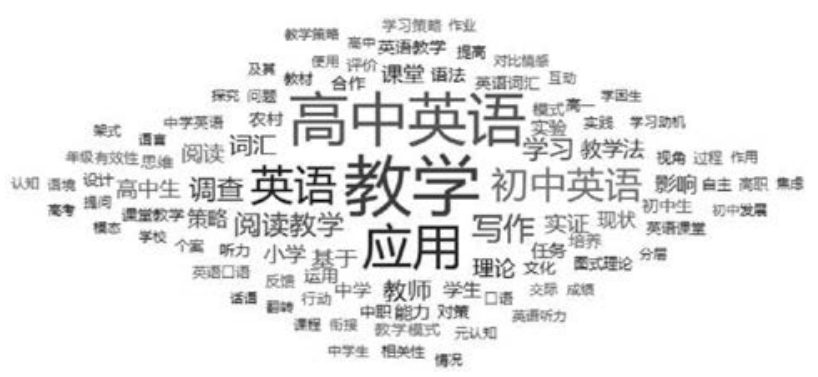

图 1 近 10 年英语教育硕士学位论文选题热点高频词词云图

首先, 从研究对象上看, 就学段来说, 高中和初中是研 究热点 “小学” 虽然也出现在词云图中，但出现频次明显 少于前两者。吴欣在对小学英语开展现状调查时发现, 小学
英语平均课时为 2.84 小时, 有的学校则仅有 1 个课时, 有 些地方对小学开设英语课程的必要性和合理性还缺乏认识, 英语课仅是一种摆设 ${ }^{[4]}$ 。同时依据《义务教育阶段英语课程 标准》 (2017 版) 对小学英语课程目标的描述，小学作为英 语学习的起始阶段, 更加关注学生积极的英语学习态度和良 好的英语学习习惯的培养, 因此知识、技能要求较低, 学习 内容比较简单, 这使许多研究生认为可供选择的课题较少。

其次, 从研究类型上看, 近 10 年英语教育硕士学位论 文开展最多的是应用性研究, 即应用相关学科领域基础研究 成果, 如互动理论、任务型教学模式等, 来解决英语教学中 的各种实际问题。开展较多的还有调查研究, 即通过对某一 现象调查，了解中国英语教学真实现状，发现问题，并提出 具有针对性的教学策略。这两类研究都是以教学实践和教学 现状为驱动的研究, 它们的广泛开展表明近 10 年英语教育 硕士学位论文选题符合《若干意见》中对专业硕士学位论文 的要求, 即“学位论文选题应来源于应用课题或现实问题, 学位论文形式可以多种多样, 可采用调研报告、应用基础研 究”。这体现了各培养单位已明确英语教育硕士以实践、应 用为研究取向的培养目标。

最后, 从研究内容上看, 近 10 年英语教育硕士学位论 文选题热点主要有教学、写作、阅读教学和学习 4 个方面。 外语教学研究不仅涉及有效的教，还涉及有效的学，因此教 学和学习都成为英语教育硕士探究的热点内容; 而对于语言 能力, 教育硕士对写作和阅读的关注度远高于听力、口语。 这一方面是因为较之于听力和口语，中国英语阅读和写作教 学研究开展早, 文献基础扎实, 且数据易采集, 另一方面则 与聚焦的学段有关系。

\section{2 基于高频词共现关系的论文选题热点分析}

挖掘高频词共现关系有助于进一步探究该学科领域研 究主题和研究方向。依据前人研究, 高频词共现关系可以表 现为两类:一类是一对高频词在多个文献中共同出现的频 次，共现频次越高，关系越紧密 ; 另一类则是某一高频词与 其他多个高频词在多个文献中共同出现的频次，共现频次越 高，该词在整个共现网络中的影响力就越大。这两类关系都 能有助于深入揭示某段时间内研究者共同关注的议题，并对 议题进行聚类分析。

图 2 为 $\mathrm{COOC}$ 软件绘制的近 10 年英语教育硕士学位选 
题热点高频词共现知识图谱，同时该软件还提供了与图谱相 对应的共现矩阵。在该图谱中，处于网络中心位置、与其他 词汇连线较多的词汇在网络中具有重要地位, 是研究者关注 的热点，而两个关键词之间连线的粗细代表高频词之间的共 现频次的高低，连线越粗，两个词之间的关系越密切。同时 从该图谱中, 还可以看到有些词聚集在一起, 形成一个主题 范畴。

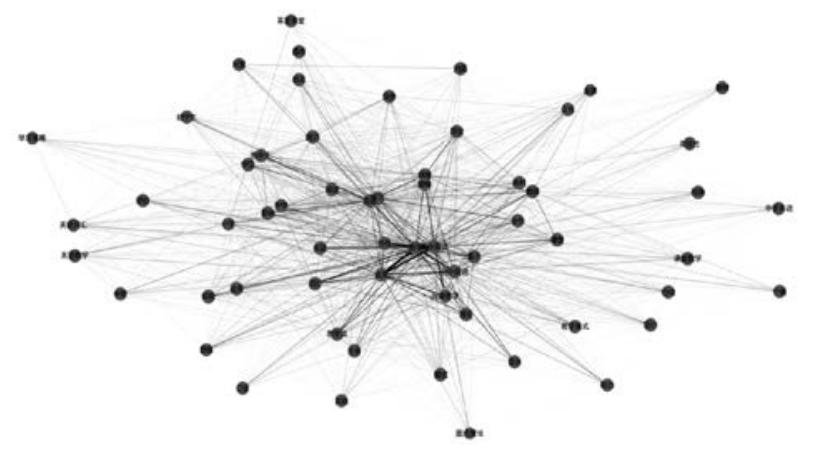

图 2 近 10 年英语教育硕士学位论文选题热点高频词共现 知识图谱

依据图 2 ，笔者发现有些词汇，如“影响”“策略”，虽 然没出现在前面提及的高频关键词里，但与其他词汇连线较 多, 共现频次较高, 说明了探究相关因素对英语教学的影响 效应以及策略在英语教学中的应用亦是近十年英语教育硕 士选题的热点。笔者进一步考察了与“影响”有高度共现关 系的词汇 发现“反馈”(69 次)、“策略”(46 次)、“任务”(37 次)、“学习动机”(37 次)、“焦虑”(31 次) 5 个指向影响因 素的词汇与“影响”有较高共现频次，表明在影响英语学习 的多种因素中，英语教育硕士对这 5 个因素的研究兴趣较高。 这一方面是受到当前各国二语习得研究趋势的影响, 即关注 教师中介、任务、学习者个体因素在二语习得中的作用，另 一方面则是顺应新课标对培养中小学生英语运用能力、自主 学习能力以及尊重学生个体特点的要求。而在与“策略” 有 高度共现关系的词汇里，笔者发现“元认知”与“策略”有 较高共现频次 (147 次), 也就是说, 出现在同一个题目中 的次数较多。元认知与策略联系密切, 这反映教育硕士对学 生英语自主学习能力的关注, 但影响英语学习的策略不只有 元认知策略一种, 因此这也一定程度上体现了策略研究的重 复性。

\section{3 基于聚类分析的论文选题热点分析}

对高频词进行聚类分析就是在共现分析的基础上，以
共现频次为分析对象，利用聚类的统计方法，把众多分析对 象之间错综复杂的共现网状关系简化为数目较少的若干类 群之间的关系并直观地表示出来的聚类的过程 ${ }^{[5]}$ 。因此采用 聚类分析有助于研究者进一步对一段时间内学科领域的研 究热点进行范畴化。笔者通过 $\mathrm{COOC}$ 软件中的聚类分析方 法对高频词进行了处理，结果如图 2 所示, 所得平均聚类系 数为 0.947 , 说明共现网络中相邻词聚集成类的程度高。

从图 2 中可以看到近 10 年英语教育硕士学位论文选题 主要聚焦于以下几个范畴：

(1)基于理论的语言技能和语言知识教学的应用性研究 对于中小学来说, 英语教学三维目标首先表现为知识和技能 目标, 因此应用先进的语言学、教育学等相关理论, 探究有 效的知识和技能教学模式成为近 10 年英语教育硕士学位论 文选题最大热点。由图 2 可知, 目前该范畴研究主要聚焦于 中学阶段, 特别是高中, 聚焦的语言技能主要为阅读、写作, 语言知识则为词汇和语法。为进一步探究英语教育硕士关注 的理论热点，笔者对题名中与理论共现的词汇进行了考察。 笔者发现，图式理论出现频次最高（228 次）, 其次是多元 智能理论 (102 次)、语境理论 (96 次)、衔接理论 (57 次)、 支架理论 (52 次) 和输入理论 (34 次)。教育部在《关于设 置和试办教育硕士专业学位的报告》中曾指出“对论文的评 价着重于考查学生综合运用所学理论和知识解决学科教学 或教育管理实际问题的能力”。上述理论的应用体现了英语 教育硕士运用所学理论解决英语教学问题的意识, 但参照近 10 年外语教学研究热点, 这些理论多缺乏前沿性, 且理论 与教学实践相结合的创新程度也不足, 重复研究现象突出。

(2)任务教学法的应用性研究及应用现状的调查研究, 自 20 世纪 80 年代以来，任务教学法随着其理论体系的不断完 善, 逐渐成为各国最受关注的外语教学范式 ${ }^{[6,7]}$, 同时高中 和义务教育阶段的新课程标准也明确提出使用任务教学法 提升学生“用语言做事情”的能力。基于这两大背景，任务 教学法成为英语教育硕士热衷探究的教学模式。探究的范式 主要有两种, 一是通过教学实验, 验证基于任务的英语教学 设计能否解决英语基础教育阶段“学用分离” 的问题 ;二是 通过问卷调查和访谈，考察当前任务教学法实施的现状及存 在的问题。

(3)英语学习自主性研究, 英语新课程标准不仅对如何 
教提出了明确要求，而且在如何学方面也明确指出 : 加强对 学生英语学习策略指导, 培养学生英语学习自主性, 为终身 学习奠定基础。因此, 从学生视角开展的研究大多聚焦于与 英语学习自主性培养相关的话题, 主要有学习策略使用现状 调查研究及学习策略应用性研究、合作学习等自主学习模式 应用性研究、自主能力培养实证研究等。

(4)相关因素影响效应实证研究, 该研究范畴主要基于 教育学、心理语言学、二语习得等领域研究成果, 以中小学 英语学习者为研究对象, 通过实证考察与二语学习相关的多 种因素的影响效应，发现导致学习者英语习得费时低效的原 因并提出相关建议或对策。考察的因素主要涉及两个维度： 学习者个体因素, 如学习策略、学习动机, 以及教师教学介 入, 如任务设计、教师反馈。

\section{4 对近 10 年英语教育硕士选题热点的反思}

对近 10 年英语教育硕士学位论文选题热点的分析表明 该阶段英语教育硕士选题符合国家对教育硕士专业培养的 要求, 主要表现为聚焦基础阶段英语教学, 侧重开展解决教 学实际问题的应用型研究而非基础性研究, 能结合教育学、 心理学和语言学等相关学科理论, 并采用科学的实证研究方 法来探究问题解决路径。但同时从该阶段选题热点中，笔者 也发现英语教育硕士学位论文选题存在明显不足。首先, 论 文选题创新性低, 存在较严重的机械照搬、复制他人研究现 象。如上面提到对学习策略的研究过度聚焦于元认知策略, 而基于理论的英语教学应用性研究则反复应用某几个理论, 且关注的问题大同小异, 如应用图式理论解决中学生阅读能 力薄弱的问题, 用语境理论解决词汇教学脱离语境的现状。 其次, 论文选题缺乏前沿性、挑战性。如对理论在英语教学 中的应用性研究, 一方面没有吸纳外语教学研究领域最新 理论成果, 另一方面没有开拓出新的研究视角, 还是局限于 传统的读写能力培养和词汇、语法知识的习得，没有真正走 进中小学英语课堂, 深入了解在新课改背景下中小学英语教 学制定了哪些新目标, 发生了哪些新变化, 出现了哪些新问 题。最后, 选题过度集中于中学, 特别是高中阶段对小学 英语教学关注度不够。二语习得研究表明, 小学开设外语 的最大好处就是充分利用儿童的可塑性和易于学好发音的 优势, 培养他们良好的学习心态和兴趣, 为深入学好外语奠 定基础 ${ }^{[8]}$ 。那么如何开设外语课来充分发挥小学生优势应是
外语教学研究中不可或缺的一部分。

针对以上不足，笔者认为培养单位需从以下两个方面改 进英语教学硕士培养方式。一方面, 实施学科基础课程和前 沿课程并重的课程设置模式, 提升学生选题的创新性和开拓 性。较之于学科基础课程, 学科前沿课程即有系统的理论基 础知识, 又涉及该学科在理论和实践领域的最新研究成果、 发展动向及存在的争议, 因此开设英语学科教学前沿课程, 可以在提升学生理论素养的同时，进一步培养学生的批判性 思维能力和创新意识, 为选择合适的学位论文研究方向提 供重要的知识与技能储备, 从而改变不断复制前人研究的 现象 ${ }^{[9]}$ 。另一方面，增强与中小学联系，建立以校内外教师 理论与实践协同指导为路径，以学生观课一授课一阅读文 献一批判性反思一发现问题一分析问题一解决问题一论文 写作为流程的教学模式，集课程学习、教学实践、课题研 究一体化，促使学生真正立足于中小学英语教学实践，用“专 家型教师”的学术敏感力寻找理论与实践相结合的契合点， 用前沿性理论和科学研究方法探究提升中小学英语教学质 量的有效路径。

\section{参考文献}

[1] 姜晶玲. 研究生学位论文质量保证措施的思考与实践 [J]. 中国 高等教育, 2005(22):37-38.

[2] 鹿秀颖, 张欣. 近十年高等教育学硕士学位论文研究热点可视 化分析 [J]. 教育信息技术,2019(7/8):98-101.

[3] 陈悦, 陈超美, 刘则渊.Citespace 知识图谱的方法论功能 [J]. 科 学学研究, 2015(2):242-253.

[4] 吴欣. 我国小学英语课程与教学改革发展的回顾与反思 [J]. 课 程．教材．教法, 2011(4):70-77.

[5] 钟伟金, 李佳, 杨兴菊. 共词分析法研究 (三): 共词聚类分析 法的原理与特点 [J]. 情报杂志 ,2008(7):118-120.

[6] Skehan,P.\&Luo,S.Developingatask-basedapproachtoassessmentina nAsiancontext[J].System,2020(90):1-15.

[7] 唐美华.“产出导向法”与“任务型教学法”比较 : 英语专业 精读课单元教学设计案例 [J]. 外语教学, 2020(1):65-69.

[8] 王初明. 外语是怎样学会的 [M]. 北京: 外语教学与研究出 版, 2010 .

[9] 林志勇, 李自然. 学科前沿课程与研究生创新实践能力培养 [J]. 高等教育研究学报, 2017(1):116-120. 